\title{
Performance Improvement of IFDMA and LFDMA using NCT Technique
}

\author{
Neeraj Pandey \\ M.Tech. Scholar \\ Department of ECE \\ Shiats-Du Allahabad,India
}

\author{
Rajiv Paulus \\ Assistant Professor \\ Department of EC \\ Shiats-Du Allahabad,India
}

\author{
A.K.Jaiswal \\ HOD \& Pro \\ Department of ECE \\ Shiats-Du Allahabad,India
}

\begin{abstract}
Orthogonal Frequency Division Multiplexing (OFDM) is a well-known modulation technique for transmission of large amount of digital data with high data rate in both wired and wireless communication systems. High peak-to-average power ratio (PAPR) is the main problem of OFDM system which can be improved by using single carrier frequency division multiple access (SC-FDMA) technique. A new scheme using LFDMA and IFDMA technique is proposed for further reducing the peak-to-average power ratio (PAPR). It transforms the SC-FDMA signal by properly choosing the transform parameters for achieving a favorable tradeoff between PAPR reduction and bit error rate (BER) performances. The simulation results show better performance of the proposed scheme than the existing technique. In this paper, we have improved the PAPR performance of single carrier frequency division multiplexing (SC-FDMA) using LFDMA and IFDMA with and without pulse shaping.
\end{abstract}

\section{Keywords}

Orthogonal frequency division multiplexing, Peak to average power ratio, Location based frequency division multiple access, interleaved frequency division multiple access.

\section{INTRODUCTION}

New Technologies and thereby new applications are emerging to provide better services over the wireless network. The success of second generation cellular system has accelerated the demand of multimedia applications availability over wireless communication systems. To achieve the goals, the next generation mobile systems are designed such that a minimum data rate of $10-20 \mathrm{Mbps}$ (at least $2 \mathrm{Mbps}$ in the moving vehicles) can be achieved in 4G. OFDM technique is suitable for multipath environment and possesses high spectral efficiency, thus it is recommended for the fourth generation cellular network. OFDM is known since a long time but it is the advancements in digital signal processing techniques which enable it to be implemented in modern wireless systems.

OFDM is a multicarrier modulation technique possessing several favourable properties like better spectral efficiency, robustness against frequency selective fading channels, higher immunity to inter symbol interference, low implementation complexity, uniform average power spectral density, very much capable to handle strong echoes and less occurrence of non-linear distortion. The PAPR of the signal $x(t)$ is given as the ratio between the peak instantaneous power and the average power occur in OFDM symbol transmission.Symbol period.

$$
\begin{gathered}
\text { PAPR }=\frac{\text { Peak power }}{\text { Average power }} \\
\text { PAPR }=\max _{0 \text { ostsT }} \frac{|x(t)|^{2}}{E\left[\mid x((t))^{2}\right]}
\end{gathered}
$$

For reduction of the high PAPR in OFDM transmission system, instantaneous peak power of the OFDM signal should be minimized. Since OFDM signal is the sum of the N signals then for highest PAPR, if we consider each signal of maximum amplitude of 1 volt and all of these signals are added up at the same time at these maximum amplitude which isunlikely but possible, making the OFDM signal amplitude of Volts which leads to $\mathrm{N}$ times higher PAPR then the PAPR of single carrier transmission system. If there is very high Peak-to-Average Power Ratio (PAPR) in OFDM based transmission systems then it requires the use of sophisticated and costly radio transmitters which have High Power Amplifiers (HPA) which can operate in a very large linear range. Otherwise non-linear signal distortion occurs, which may lead to high adjacent channel interference and performance degradation. These demands result in complex and costly hardware systems. Hence to minimize the problem of complex hardware design it is better to employ efficient PAPR reduction techniques.

There are different PAPR reduction techniques and their selection criteria which are given in the literature (Han et al 2005) (Vijayaranganet al 2009). Single carrier frequency division multiple access (SC-FDMA) is the technique which basically uses the single carrier modulation, which is an extension of the OFDMA system having similar overall complexity and performance. It has a major advantage over OFDMA system that it has lower PAPR because of its integral single carrier structure

(Falconeret al 2002) and due to its high transmission power efficiency. It is widely used in uplink multiple access transmission of 3G LTE of 3GPP [4].

In OFDM system to reduce the PAPR,another type of companding technique is used, known as Nonlinear Companding Transform. This technique converts the OFDM signals into a specific statistics form, which can be given by a piecewise probability density function with an inflexion point. Theoretical analysis shows that, this scheme may drastically decrease the impact of companding distortion on the BER performance to achieve a given PAPR level, while simultaneously achieving a favorable trade-off between the PAPR reductions. 
To improve the problem of PAPR in OFDM system the technique of interleaved frequency division multiple access (IFDMA) and location based frequency division multiple access (LFDMA) technique is employed in SCFDMA.Single carrier frequency division multiple access (SC-FDMA) is the technique which basically uses the single carrier modulation, is an extension of the OFDMA system. In SCFDMA due to its nature of single carrier modulation it gives less PAPR compared to OFDM system.

\section{METHODOLOGY}

\subsection{Proposed PAPR reduction scheme}

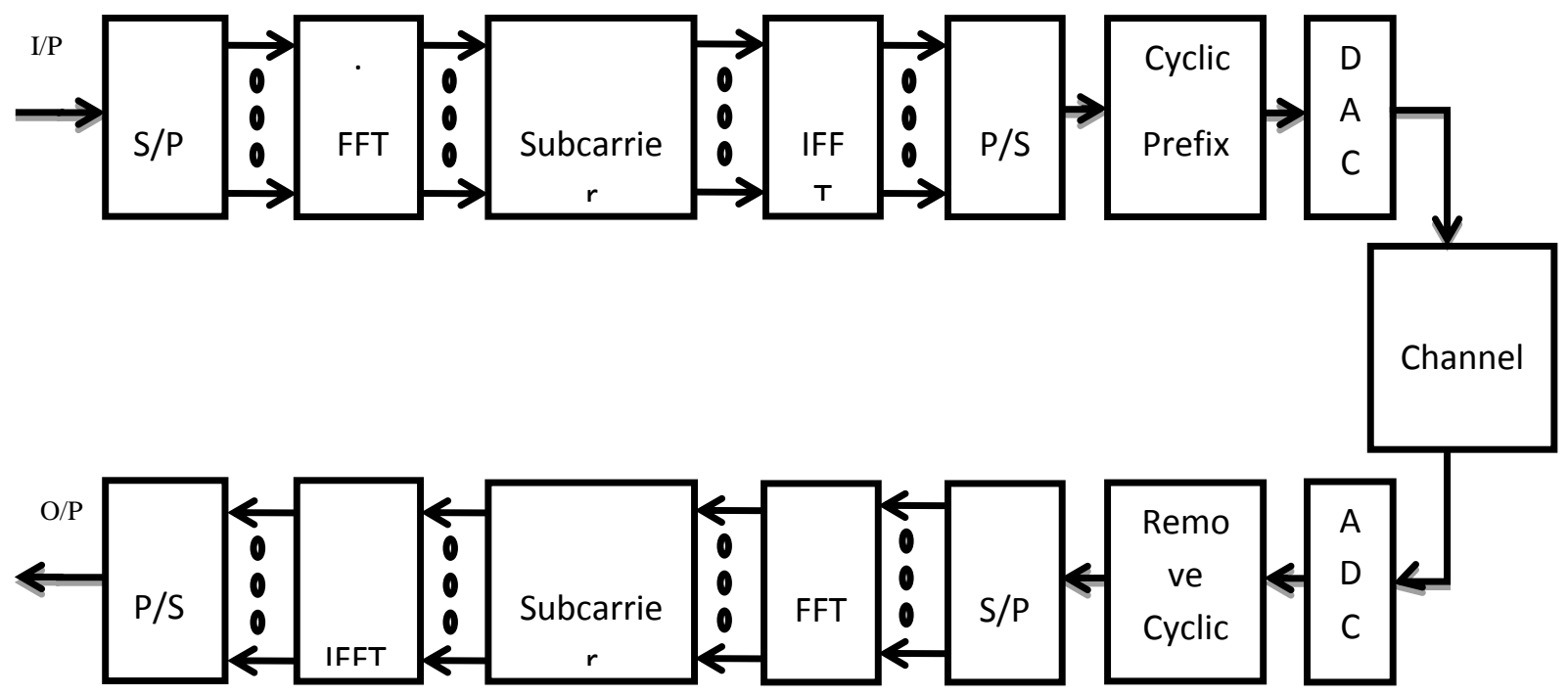

Fig.1 Block diagram of proposed scheme

The above figure 1 shows the block diagram of the proposed PAPR reduction technique of the SC-FDMA and nonlinear companding technique. SC-FDMA is a discrete Fourier transform-spread version of OFDMA, where time domain data symbols are converted to frequency domain by DFT before applying OFDMA modulation. By applying the DFT before passing it through the IDFT it ensures that the subcarriers are orthogonal to each other which results the overall transmit signal as the single carrier signal leading to the single carrier frequency division multiple access (SCFDMA). To decrease the computational complexity of the system, FFT is used in place of DFT operation and IFFT in place of IDFT operation. In the subcarrier mapping scheme there are $\mathrm{M}$ subcarriers, among which $\mathrm{N}$ subcarriers are placed by the input data where $\mathrm{M}<\mathrm{N}$.

There are two modes to choose the subcarriers for transmission in SC-FDMA. In first mode the Located FDMA (LFDMA), FFT outputs of the input data are placed on the consecutive subcarriers in the form of chunk. While in the second mode of subcarrier mapping the distributed FDMA, the FFT outputs of the input data are placed on the entire subcarriers with zeros occupying in unused subcarriers. There is another case of distributed FDMA where FFT outputs of the input data are placed over the entire subcarriers having the equal distance in between each subcarrier,known as interleaved FDMA (IFDMA) (Sorgeret al 1998).

In first type the Located FDMA (LFDMA), FFT outputs of the input data are placed on the consecutive subcarriers in the form of chunk

These two subcarrier mapping schemes have advantages over each other as Distributed FDMA has simpler scheduling and higher frequency diversity while localized FDMA has higher frequency selective gain(Houet al 2007).
Let $x_{m}$ be the data symbols which is to be modulated,on doing the FFT of $x_{m}$ the frequency domain samples are $X_{m}$. Let $X_{n}$ be the frequency domain samples after the subcarrier mapping of the frequency domain samples $X_{m}$ and the time domain samples after taking the IFFT of the $X_{n}$ be $x_{n}$.

For LFDMA the frequency domain samples $X_{n}$ can be given as following,

$$
X_{n=Q . m}=\left\{\begin{array}{cc}
X_{m}, & 0 \leq n \leq M-1 \\
0 & M \leq n \leq N-1
\end{array}\right.
$$

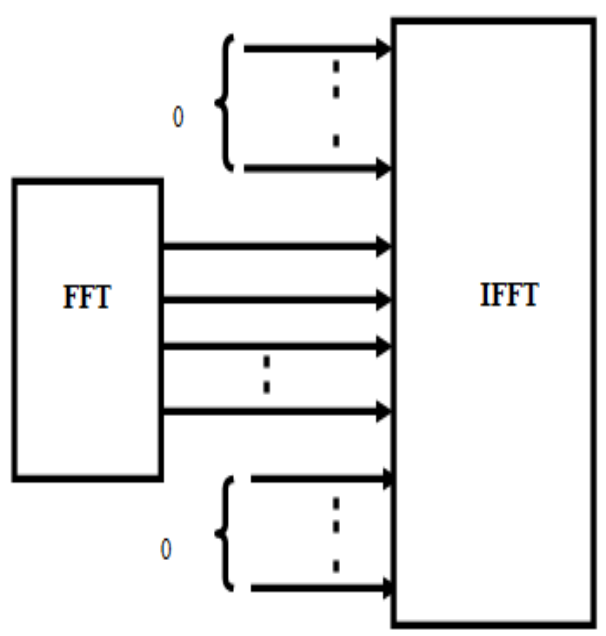

Fig.2LFDMA sub-carrier mapping

For IFDMA the frequency domain samples $X_{n}$ can be given as following, 


$$
X_{n=Q . m}=\left\{\begin{array}{cr}
X_{m}, & 0 \leq m \leq M-1 \\
0 & \text { otherwise }
\end{array}\right.
$$

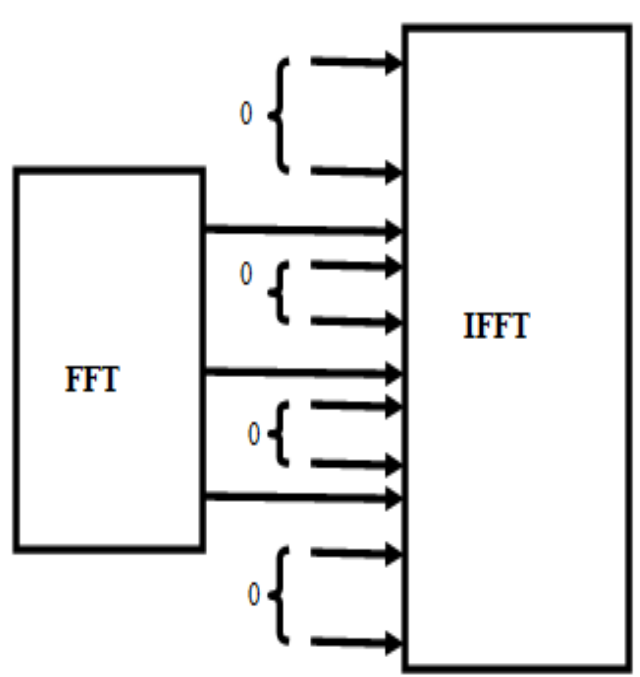

Fig3IFDMA sub-carrier mapping

After taking the IFFT of the frequency domain subcarrier mapped signal $X_{n}$ we will get the time domain

samples as $x_{n}$.now we will transform the signal $x_{n}$ according to the companding transform function $f($.$) .$ The transformed signal can be given as following,

$$
y_{n=} f\left(x_{n}\right), \quad n=0,1,2, \ldots \ldots \ldots N-1
$$

The transform parameters such as $\mathrm{k}$ and $\mathrm{p}$ are variables which are used to obtain ultimate companding form so that we can achivefavourble tradeoff between reduced PAPR and bit error rate (BER) perform.

The transfer curves is shown in Figure.4. It can be seen from Fig. that this scheme compresses the large signals while partially expands the small ones simultaneously. This transform has some other advantages such as it can maintain a constant average power level in the transformed signal. As a result, it not only reduces the PAPR more effectively, but also the small signals become more immune from the channel noise.

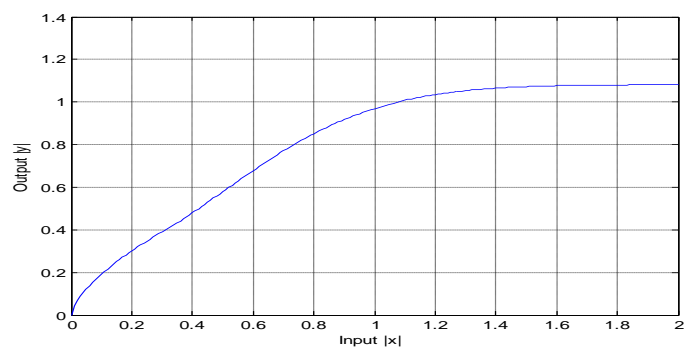

Figure.4 Transfer curve of the NCT

\section{SIMULATION DESIGN}

In our simulation model we have taken the total number of subcarriers as 512, input data block size to 16 and bandwidth spreading factor to 32. To acquire the CCDF of PAPR to be ten thousand times, a uniformly random data points were generated. Transmission bandwidth of $5 \mathrm{MHz}$ and symbol constellations as QPSK was considered. Show in table 1.
In our simulation model we have applied the raised-cosine (RC) pulse and squared-root raised-cosine (RRC) pulse [11] which is widely used for pulse shaping in wireless communications.

Table 1.simulation parameter

\begin{tabular}{|l|l|}
\hline parameter & value \\
\hline Sub carrier(N) & 512 \\
\hline $\begin{array}{l}\text { Bandwidth } \\
\text { spreading } \\
\text { factor }\end{array}$ & 32 \\
\hline $\begin{array}{l}\text { Transmission } \\
\text { bandwidth }\end{array}$ & $5 \mathrm{MHz}$ \\
\hline $\begin{array}{l}\text { Input data } \\
\text { block size }\end{array}$ & 16 \\
\hline $\begin{array}{l}\text { Modulation } \\
\text { technique }\end{array}$ & QAM \\
\hline
\end{tabular}

\section{RESULT AND DISCUSSION}

The performance of the proposed scheme is evaluated in terms of the reduction of the PAPR and BER performance through numerical simulations. PAPR statics are shown in terms of CCDF (Complementary Cumulative Distribution Function) which gives the probability that PAPR is greater than a certain PAPR value PAPR0 ( $\operatorname{Pr}\{P A P R>P A P R 0\})$. So we have compared the performance of OFDMA and the proposed scheme.

Figure 5 shows CCDF plots of PAPR for the OFDMA of QPSK modulation with pulse shaping. From fig, PAPR probability approximately $.0001 \%$ for $10.2 \mathrm{~dB}$ whereas probability of arising PAPR for $8.5 \mathrm{~dB}$ is 0.01 .

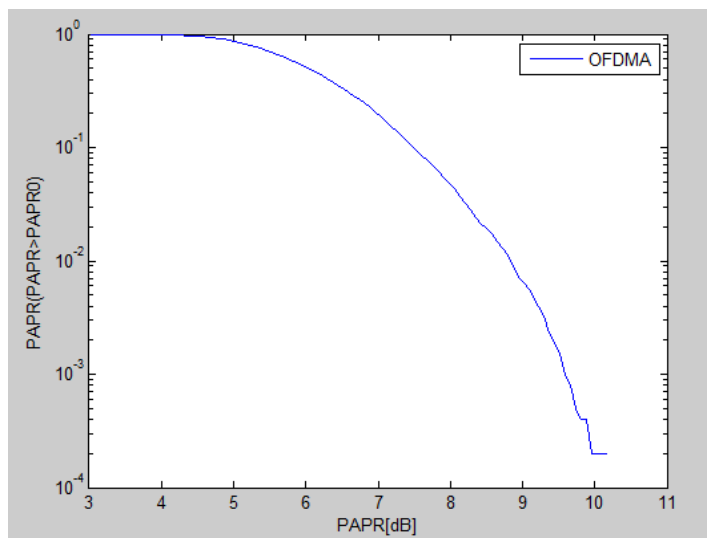

Fig.5CCDF plot of PAPR for OFDMA with pulse shaping

Fig 6 show CCDF plot of OFDMA.DFDMA, LFDMA and IFDMA without pulse shaping .probability of arising PAPR for IFDMA has 0db which is an ideal case whereas .0001\% probability of arising PAPR for OFDMA ,DFDMA and LFDMA respectively $10.2 \mathrm{db}, 6.8 \mathrm{db}$ and $6.7 \mathrm{db}$. 


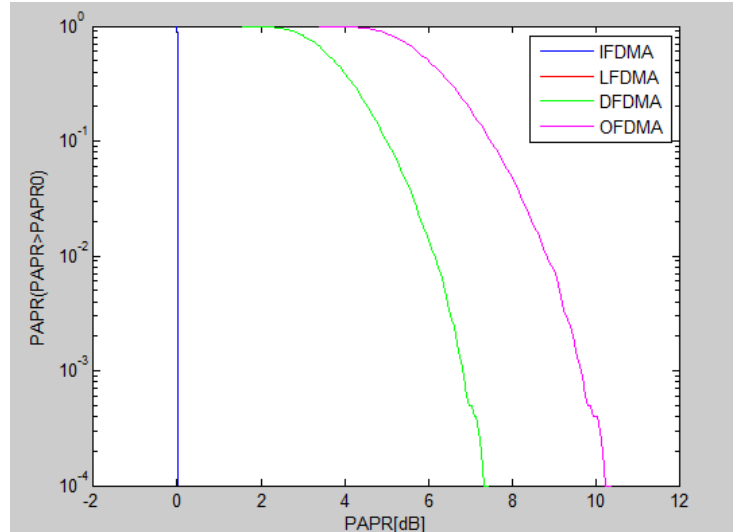

Fig 6CCDF plot of PAPR for OFDMA.LFDMA, DFDMA and IFDMA without pulse shaping.

\section{CONCLUSION}

Aim of this paper is to improve the PAPR performance of SCFDMA system using LFDMA and IFDMA sub-carrier mapping furthermore compared the PAPR performance of the system with and without Pulse shaping. It is shown that IFDMA and LFDMA signals have lower PAPR compared OFDMA. Also, we have shown that LFDMA incurs higher PAPR compared to IFDMA, but compared to OFDMA, it is lower, though not significantly, without degrading the BER performance. Another noticeable fact is that pulse shaping increases PAPR and that roll off factor in the case of raisedcosine pulse shaping has a significant impact on PAPR of IFDMA. A pulse shaping filter should be designed carefully in order to reduce the PAPR without degrading the system performance. Hence overall performance of proposed scheme is good in comparison to OFDMA.

\section{ACKNOWLEDGEMENTS}

I would like to thank my advisor Dr Rajiv paulus assistant professor SSET Sam higgingbottom institute of agriculture technology and sciences (deemed-to-be university), Allahabad, for providing guidance and all require facilities and thank to my co- advisor prof A.K. Jaiswal HOD of ECE, SSET, for their constant co-operation, help, guidance and support during project period.

\section{REFERENCES}

[1] S. H. Han and J. H. Lee, "An Overview of Peak-toAverage Power Ratio Reduction Techniques for Multicarrier Transmission," IEEE Wireless Communication, vol. 12, no. 2, April 2005, pp. 56-65.

[2] V. Vijayarangan, Dr. (Mrs) R. Sukanesh , “An Overview Of Techniques For Reducing Peak to Average Power Ratio And Its Selection Criteria For Orthogonal Frequency Division multiplexing Radio Systems" Journal Of Theoretical And Applied Information Technology, Year 2009 ,Vol-5, No-5, EIssn- 1817-3195/Issn-1992-8645.

[3] D.Falconer, S. L. Ariyavisitakul, A. Benyamin-Seeyar and B. Eidson, "Frequency Domain Equalization for Single-Carrier Broadband Wireless Systems," IEEE Communcation. Mag., vol. 40, no.4, April 2002, pp. 5866.

[4] 3rd Generation Partnership Project (3GPP); Technical Specification Group Radio Access Network; Physical Layer Aspects for Evolved UTRA, http://www.3gpp.org/ftp/Specs/html-info/25814.htm

[5] U. Sorger, I. De Broeck and M. Schnell, "Interleaved FDMA - A New Spread-Spectrum Multiple-Access Scheme," Proc. IEEE ICC '98, pp. 1013-1017, Atlanta, GA, June 1998.

[6] T. S. Rappaport, Wireless Communications: Principles and Practice, Second Edition. Prentice Hall, 2002.

[7] Hou, J., Ge, J., Zhai, D., \& Li, J. (2010). Peak-to-average power ratio reduction of OFDM signals with nonlinear companding scheme. IEEE Transactions on Broadcasting,56(2),258-262. 